\title{
COMPRENSIÓN LECTORA EN LOS DOCENTES DE EDUCACIÓN BÁSICA DE LA REGIÓN JUNIÍN
}

\author{
READER COMPREHENSION IN THE BASIC EDUCATION TEACHERS JUNÍN \\ REGION \\ Luis Yarlequé Ch'., Leda Javier A., Edith Nuñez Ll., Linda Navarro G., Alberto Cerrón L., Julie Monroe A. \\ Universidad Nacional del Centro del Perú \\ (RECIBIDO 4/2/2012; ACEPTADo el 18/6/2012)
}

\begin{abstract}
RESUMEN
La investigación que se presenta es de tipo aplicada y de nivel descriptivo. En ella se estudió la comprensión lectora de los docentes de los tres niveles de educación básica de la región Junín. Se empleó el método descriptivo con un diseño descriptivo comparativo, se tuvo una muestra de 357 sujetos a los que se aplicó una prueba de compresión lectora desarrollada para efectos de la investigación. Los resultados permitieron probar entre otras cosas que el nivel de enseñanza el que trabajan los docentes no le otorgan ventajas o desventajas significativas en su comprensión lectora, así mismo que las variables género, situación laboral, institución en la que desarrolló sus estudios profesionales e incluso los estudios de posgrado, no tienen un papel distintivo en la comprensión lectora de los docentes.
\end{abstract}

Palabras clave: Comprensión lectora

\begin{abstract}
The investigation that turns up belongs to fellow once was applied and of descriptive level. The region's reader of the teachers of the 3 basic education levels studied understanding herself In her Junín. He used the descriptive method with a descriptive design comparative, one had a 357- subjects sign whom applied a compression test reader once was developed in order to the investigation's properties itself.

Aftermaths permitted testing among other things that the basic education level whereon operates the teachers reader does not grant him perks or significant disadvantages in her understanding, likewise than the variables kind, labor situation, institution that I develop his professional studies in and enclosure the postgrade studies, they do not have a distinctive paper in the understanding the teachers's reader
\end{abstract}

Keywords: reading comprehension 


\section{INTRODUCCIÓN}

La comprensión lectora enfocada desde el punto de vista del desarrollo cultural de las sociedades es hoy en día en el Perú uno de los problemas más urgentes por solucionar en el ámbito educativo. Ello implica, no solo hablar de la evaluación de los estudiantes de educación básica de los diferentes niveles, sino también de los docentes, quienes son los encargados de transmitir los conocimientos y ponerlos en acción a través del uso de las diversas estrategias.

De ello se puede inferir entonces que el maestro se convierte en el modelo de sus estudiantes, por eso "un buen maestro" debería hablar y escribir bien, porque los estudiantes muchas veces son el vivo reflejo de sus profesores, sin soslayar la labor educativa que cumplen algunos padres de familia para con sus hijos.

Así se hace necesario involucrar directamente a las personas que tienen que ver con el problema, entendiéndose que se pueden encontrar profesionales de la educación en los distintos niveles, llámese educación inicial, primaria y secundaria. Y se puede preguntar si ¿son los profesionales de la educación culpables del problema de comprensión lectora de los estudiantes de diferentes niveles? Ahora bien, Vega (2011); Rondón (2010); Mesías (2008); Aquino y Ríos (2007); Cubas (2007); Correa (2005); Mendoza (2004); Arrieta y Meza (2002); Aliaga (2000); Tapia (1995); Rojo (1990); Paris, Cross y Lipson (1984) han investigado respecto al tema en estudio. Por otro lado, se tiene el reporte presentado por los evaluadores de la prueba PISA, en donde se indica que el Perú se encuentra en el penúltimo lugar en comprensión lectora. Pero, no se han registrado trabajos en esta dirección en la Región Junín.

Es en este contexto se abordó el siguiente problema: ¿Existen diferencias en la comprensión lectora de los docentes de los tres niveles de educación básica de la región Junín? Correspondiente con ello, el objetivo general de la investigación fue determinar si existen diferencias en la comprensión lectora en los docentes de los tres niveles de educación básica de la región Junín. Y los objetivos específicos fueron: A) Evaluar la compresión lectora en los docentes de los tres niveles de educación básica de la región Junín y B) Comparar la comprensión lectora en los docentes de los tres niveles de educación básica de la región Junín.

Las hipótesis planteadas sostenían que, existen diferencias en la comprensión lectora en los docentes de los tres niveles de educación básica de la región Junín. Y la hipótesis específica afirmaba que los profesores de educación secundaria presentan mayor nivel de comprensión lectora que los de primaria y estos que los de inicial.

El trabajo en cuestión se justifica en que se ha notado ciertas deficiencias en la comprensión lectora de los docentes -así lo muestran las observaciones empíricas- donde los docentes de educación básica no tienen predilección por la lectura y los que leen, no comprenden el texto. Ahora bien, si un profesor no comprende lo que lee, ¿cómo es que pueden enseñar a los estudiantes a comprender? Por eso que trabajó en torno a este tema. 


\section{MÉTODO}

\section{Tipo y nivel de investigación}

La investigación es de tipo aplicada y de nivel descriptivo. (Sánchez y Reyes, 2006).

\section{Método y diseño}

El método empleado fue el descriptivo (Alarcón 1991) con un diseño descriptivo comparativo (Sánchez y Reyes, 2006) cuyo esquema es el siguiente:

Diseño descriptivo comparativo

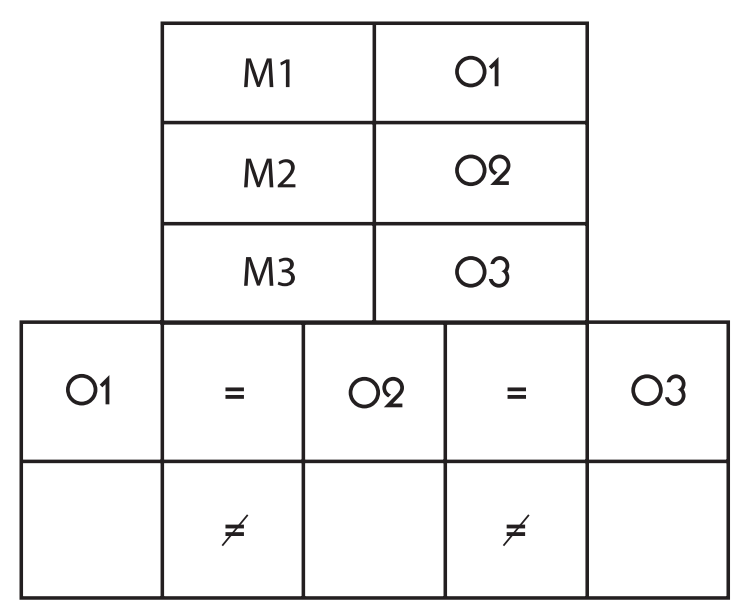

Donde M1, M2 y M3 representan a las muestras de docentes de educación básica de los tres niveles respectivamente. $\mathrm{O} 1, \mathrm{O} 2$ y $\mathrm{O} 3$ son las observaciones en cada una de las muestras.

\section{Población y muestra}

La población estuvo constituida por el total de docentes de los tres niveles de educación básica de la Región Junín.

La muestra estuvo conformada por 357 docentes capacitados a través del convenio de la Universidad Nacional del Centro del Perú con el Ministerio de Educación; así como por capacitadores, también participaron docentes maestristas y docentes pertenecientes al Programa de Complementación Académica y Licenciatura. Todos ellos de las diversas áreas y niveles de educación, de ambos sexos, procedentes de universidades e institutos pedagógicos, nombrados y contratados, cuyos tiempos de servicio van desde menos de un año a más de 26 . La muestra fue seleccionada en forma no probabilística.

\section{Técnicas e instrumentos de la investigación}

Las técnicas empleadas fueron la observación indirecta (Yarlequé, Javier y Monroe, 2002) así mismo la técnica sicométrica. El instrumento fundamental fue la 
prueba de compresión lectora desarrollada para efectos de la investigación. Para la validación del instrumento se realizó el siguiente proceso: 1) Se tomó una lectura de José Martí y se elaboraron los ítems, teniendo en cuenta preguntas que pusieran de manifiesto el nivel literal, inferencial y criterial, asimismo fenómenos cognitivos como la memoria, percepción y pensamiento y procesos como: el reconocimiento, la reconstrucción, análisis, síntesis, comprensión, deducción, abstracción y evaluación, para lo cual se elaboró una tabla de especificaciones para verificar la validez de contenido. 2) Esta prueba se aplicó a 150 docentes con el propósito de hacer el ajuste semántico de los ítems. Los resultados indicaron que dichos ítems eran comprendidos por los docentes; por tanto el instrumento goza de validez semántica. 3) La prueba elaborada y reajustada fue aplicada a un grupo piloto de 30 sujetos con la finalidad de darle validez estadística a través de la correlación de cada ítem con toda la prueba. La prueba en general tiene un coeficiente de validez igual a 4.43 .

\section{Técnicas de procesamiento de datos}

Para el procesamiento de datos se emplearon medidas de tendencia central (media aritmética), de dispersión (desviación estándar) tablas de frecuencia y porcentajes. Para las correlaciones se utilizó la $\mathrm{r}$ de Pearson y para la contrastación de las hipótesis se usó la prueba Z.

\section{RESULTADOS}

Al dar una mirada a los resultados de los docentes en función de la institución en la que laboran referidos a los niveles de comprensión: literal, inferencial y criterial, los egresados de las universidades, tienen medias ligeramente más altas con respecto a los de los institutos. En los fenómenos psíquicos ocurre lo mismo en todos los casos, con respecto a los procesos cognitivos, en la mayoría de los casos los egresados de las universidades tienen medias ligeramente más altas que de los institutos, excepto en los procesos cognitivos de reconstrucción y evaluación, en donde las medias más altas corresponden a los de los institutos. En toda la prueba, son los de las universidades que obtienen la media general ligeramente más alta con respecto a los de los institutos. En cuanto los resultados de los docentes en función al nivel en el que trabajan, muestran que los docentes que laboran en el nivel secundario presentan la media más alta en el nivel literal y la más baja los de inicial. Por el contrario, en el nivel inferencial son los de inicial los que presentan la media más alta. Curiosamente en el nivel criterial son los de primaria. Analizando los resultados en función de los fenómenos psíquicos, presentan mejor performance en memoria los que trabajan en secundaria, esto se repite cuando se evalúa el pensamiento. No obstante, en percepción la media más alta la ostentan los que trabajan en educación inicial. Analizando cada una de las habilidades cognitivas, se encuentra que los profesores que laboran en secundaria presentan promedios más altos en reconocimiento, deducción y abstracción. Por su parte los que trabajan en primaria, alcanzan las medias más altas en comprensión, reconstrucción y evaluación. Los de educación inicial únicamente superan a los demás en síntesis. Aunque también en el puntaje general. 
Así mismo los resultados de la muestra en función de los estudios realizados muestran que los ítems que exploran respuestas de nivel literal e inferencial, en ambos casos la media ligeramente más alta la ostentan los que no poseen estudio de posgrado. En el nivel criterial destacan los docentes con estudios de posgrado. Observando los resultados por fenómenos psíquicos, en memoria, pensamiento y percepción las medias más altas corresponden a los que no tienen estudios de posgrado. En el proceso de análisis, se ha encontrado que los que no tienen estudios de posgrado mantienen medias más ligeramente altas en reconocimiento, deducción, síntesis y evaluación. Mientras que los que los tienen superan a los otros sub grupos en comprensión, abstracción y reconstrucción, siendo la media general más alta la de los que no tienen estudios de posgrado.

\section{Contrastación de hipótesis}

$\mathbf{H}_{\mathbf{0}}$ No existen diferencias significativas entre las medias de los docentes del nivel secundarios, con respecto a los de primaria en la prueba de comprensión lectora.

$\mathbf{H}_{1}$ Si existen diferencias significativas entre las medias de los docentes del nivel secundarios, con respecto a los de primaria en la prueba de comprensión lectora.

$\mathbf{H}_{\mathbf{0}} \quad$ No existen diferencias significativas entre las medias de los docentes del nivel secundarios, con respecto a los de inicial en la prueba de comprensión lectora.

$\mathbf{H}_{2} \quad \mathrm{Si}$ existen diferencias significativas entre las medias de los docentes del nivel secundarios, con respecto a los de inicial en la prueba de comprensión lectora.

$\mathbf{H}_{\mathbf{0}} \quad$ No existen diferencias significativas entre las medias de los docentes del nivel primario, con respecto a los de inicial en la prueba de comprensión lectora.

$\mathbf{H}_{3} \quad \mathbf{S i}$ existen diferencias significativas entre las medias de los docentes del nivel primario, con respecto a los de inicial en la prueba de comprensión lectora.

Tabla 1: Comparación de medias entre docentes de inicial, primaria y secundaria en la prueba de comprensión lectora

\begin{tabular}{|c|c|c|c|c|c|c|}
\hline \multicolumn{2}{|c|}{ Medias } & \multirow{2}{*}{ gl } & \multirow{2}{*}{$\mathrm{r}$} & \multirow{2}{*}{$\mathrm{zt}$} & \multirow{2}{*}{$\mathrm{zp}$} & \multirow{2}{*}{ Diagnóstico } \\
\hline secundaria & primaria & & & & & \\
\hline 12.25 & 12.25 & 337 & 0.05 & 1,96 & 0.49 & No significativo \\
\hline secundaria & inicial & & & & & \\
\hline 12.25 & 12.27 & 237 & 0.05 & 1,96 & 0.94 & No significativo \\
\hline primaria & inicial & & & & & \\
\hline 12.25 & 12.27 & 134 & 0.05 & 1,96 & 0.73 & No significativo \\
\hline
\end{tabular}

En la Tabla 1, se observa la comparación de los subgrupos por pares, estos resultados llevados a la prueba $\mathrm{z}$, muestran que no existen diferencias significativas en ninguno de los casos. Con lo que se aceptan las hipótesis nulas y se rechazan las hipótesis alternas en cada caso. 


\section{DISCUSIÓN}

Los resultados presentados han mostrado que no existen diferencias significativas entre los puntajes alcanzados por los docentes que han estudiado en universidades y los de institutos en la prueba de comprensión lectora, pese a que los docentes procedentes de universidades obtienen medias ligeramente superiores en varios aspectos de la prueba. Esto podría significar que la universidad no otorga a sus estudiantes ventajas significativas con respecto a los institutos, por lo menos en lo que a la capacidad de comprensión lectora se refiere. Estos resultados tienen relación con los de Arrieta y Meza (2002) en Venezuela. Ahora bien, en otro contexto, simplemente podría poner a la luz las virtudes y ventajas de los institutos. No obstante, ya que los docentes de institutos han obtenido una media superior a 10,5 entonces puede decirse que aunque en términos fórmales estarían aprobados, los calificativos no son los deseables considerando que se trata de educadores y que el Ministerio de Educación considera 14 como mínima nota aprobatoria. Algo análogo se puede decir con respecto a la media de docentes que proceden de universidades. En realidad ambos grupos han obtenido medias por debajo de lo deseable en profesionales que tienen la tarea de formar a los varones y mujeres de la región Junín.

Otro aspecto interesante para analizar es la comparación entre los resultados obtenidos por las mujeres y varones de la muestra, en la prueba de comprensión lectora. También aquí, pese a que las docentes obtienen medias superiores a sus análogos varones en casi todo los casos, esta aparente superioridad no alcanza el nivel de significación, por consiguiente es legítimo señalar que por lo menos a la luz del presente estudio, el género no tendría un papel distintivo en la comprensión lectora de los docentes. Y aquí nuevamente se ha observado que la media de ambos sub grupos, aunque está por encima del 10,5 se encuentra por debajo del 14.

Por otro lado, al analizar los resultados por niveles se ha observado que el trabajar en uno u otro nivel de la educación básica, no otorga al docente, ventajas o desventajas en comprensión lectora. En efecto, cuando se hacen las comparaciones por pares de los docentes que laboran en los niveles de inicial, primaria y secundaria, en ningún caso se hallaron diferencias significativas. No obstante, es pertinente detenerse un poco en las particularidades de estos resultados; los tres grupos no solo presentan medias muy similares, sino que no alcanzan el tercio superior de la distribución y si tomásemos en cuenta el límite tradicional aprobados desaprobados $(10,5)$, se encontrarían en el sector de aprobados, pero es notable que desde la perspectiva del Ministerio de Educación ningún subgrupo estaría aprobado. Es notable también, que los profesores que laboran en el nivel secundario tienden a obtener mejores resultados frente a la prueba en los ítems que exploran memoria y pensamiento, mientras que los de inicial tienden a orientarse mejor en los ítems que exploran percepción. Ahora bien, la exploración proceso por proceso permite comprender que, aunque los docentes no se diferencian en sus puntajes promedio, sí difieren entre sí en los procesos involucrados en la resolución de las tareas propias de la prueba. Esto probablemente se relaciona con el concepto 
de estrategia cognitiva (Gagné, 1979), según el cual, cada individuo se organiza de un modo especial para enfrentar con éxito las tareas que se le imponen. De tal suerte que aunque los resultados sean cuantitativamente similares, de ello no puede deducirse similitud en el modo de abordar las tareas ni en los procesos cognitivos involucrados.

Analizados los resultados en función de la situación laboral del docente, se ha encontrado que esta variable parece ser írrita. En efecto, las comparaciones de medias muestran que los contratados, e incluso los desempleados, no tienen desventajas significativas con respecto a los nombrados en la prueba de comprensión lectora, así pues, frases como; "sí alcanzo un nombramiento podré rendir más y mejor, porque tendré seguridad económica" o "los contratados rinden más y mejor que los nombrados, ya que se esfuerzan por obtener seguridad laboral" parecen no tener asidero científico por lo menos en la variable estudiada. Es posible, sin embargo, que algunos casos puntuales hayan conducido a formular tales aseveraciones. Nuestros resultados brindan argumentos por lo menos para mantener una legítima duda al respecto.

Hay en este punto un dato importante que apreciar, pese a no haber diferencias significativas entre los tres subgrupos, existe una tendencia decreciente notable en las medias desde los nombrados hasta los desempleados, pasando por los contratados, sería necesario hacer estudios complementarios al presente, pues de comprobarse rigurosamente, este fenómeno en la realidad, podría estar ocurriendo que el desarrollo de la actividad docente otorgue al sujeto cierta ventaja, con respecto al que no la desarrolla o lo hace por corto tiempo, en su capacidad de comprensión lectora. Este fenómeno respondería a las leyes del desarrollo cognitivo, según una de las cuales el ejercicio de la función conduce al desarrollo del fenómeno (Vygotski,1988).

Ocupan un lugar especial en esta discusión, los hallazgos al comparar la comprensión lectora de quienes realizaron estudios de posgrado, quienes realizaron otros estudios que no son de posgrado y quienes no tienen estudio adicional alguno (al margen de los estudios que los condujeron a optar el título para la docencia). Es esperado, y además deseable, que no existan diferencias en la comprensión lectora al comparar a los docentes en función del nivel en que trabajan. Es igualmente deseable que no se diferencien en virtud del género. Es beneficioso para los institutos que sus docentes no tengan desventajas significativas con respecto a los que se formaron en la universidad. Incluso es comprensible aunque no deseable, que los profesores nombrados no muestren ventajas ostensibles con respecto a los contratados y desempleados. Pero es preocupante que los estudios de posgrado no estén marcando diferencias en una capacidad tan importante como la comprensión lectora con respecto a quienes no han hecho estudios de posgrado, incluso con respecto a los que no han hecho estudio alguno, al margen de los que le permitieron optar el título. Estos resultados deben entenderse como una seria llamada de atención a las escuelas de posgrado, más aún si se observa las medias de los tres subgrupos en mención. 
En este punto nuestros hallazgos vuelven a relacionarse con los de Arrieta y Meza (2002), quienes llegaron a encontrar casos de analfabetismo funcional en estudiantes universitarios de Venezuela. Una de las cosas que más preocupó a los investigadores fue precisamente el hecho de que el analfabetismo funcional se detectó en estudiantes de educación. También Aquino y rios (2007) detectaron dificultades en el nivel de extrapolación, interpretación y traducción, en estudiantes de educación.

Es posible que el lector acucioso pueda argüir que los 21 puntos de la prueba, convertidos al sistema vigesimal muestran que los docentes han obtenido notas por encima del 10,5 y probablemente más de uno señalaría que son resultados mejores de los que ha señalado el Ministerio de Educación (Junio,2007). Sin embargo, tratándose de los profesionales que forman a los niños, adolescentes y jóvenes de la región, las notas promedio alcanzadas no son las deseables. Se hace necesario pues diseñar y experimentar programas para favorecer el desarrollo de la comprensión lectora de adultos. Debe recordarse que una serie de trabajos como los de Nicholson (1980), Raim (1980), Milicia (1983), Rojo (1990) y Tapia (1995), entre otros, han probado que se puede intervenir positivamente sobre la comprensión lectora de niños, sin embargo, no se conocen experimentos con adultos. Así, se hace ineludible enfrentar el problema descrito, ya que es razonable suponer que las deficiencias en la comprensión lectora, puestas de manifiesto en los estudiantes de educación básica del Perú (PISA 2000), (citada en Educación y Ciencia 2008), se relacionan con los hallazgos aquí expuestos. Ya que si el docente no ha desarrollado su comprensión lectora en los niveles deseables, es poco probable que logre el desarrollo de la compresión lectora de sus estudiantes. Quedan, pues, planteados un conjunto de problemas para los investigadores interesados en tan fascinante temática.

\section{CONCLUSIONES}

Los hallazgos aquí expuestos permiten concluir que el nivel (inicial, primaria y secundaria) no tendría un efecto distintivo sobre la comprensión lectora de los docentes.

El género, la situación laboral y la institución en la que se formó el docente son también variables írritas en lo que a la compresión lectora se refiere. Por consiguiente, no puede esperarse ventajas o desventajas a partir de dichas variables.

Los docentes que han realizado estudios de posgrado no presentan mayor desarrollo de la comprensión lectora respecto de los que no los hicieron; en consecuencia, los datos aquí expuestos, permiten suponer que los estudios de posgrado, tal y como se vienen realizando, no estarían contribuyendo al desarrollo de tan importante capacidad en los docentes de la región Junín.

Se ha diseñado y validado una prueba corta y de sencilla aplicación que permite evaluar la comprensión lectora de los docentes, distinguiendo sus respuestas ante preguntas que surgen respuestas literales, inferenciales y criteriales. Así mismo, la prueba permite distinguir el fenómeno y los procesos cognitivos puestas en acción 
en cada caso. Tal instrumento puede ser utilizado en la región, por lo menos en los casos en que se tenga que hacer una aplicación colectiva y rápida.

\section{Nota de reconocimiento}

Al equipo de jóvenes investigadores: Cerrón L. Alberto; Javier A. Leda; Monroe A. Julie; Nuñez LL. Edith; Navarro G. Linda, que se esfuerzan por contribuir en el avance de la ciencia y de la educación.

\section{REFERENCIAS BIBLIOGRÁFICAS}

Alarcón, R. (1991). Métodos y diseños de investigación del comportamiento. Lima: Universidad Peruana Cayetano Heredia.

Aquino, P. y Rios. Z (2007). "Niveles de comprensión lectora y su relación con la resolución de problemas matemáticos". Revista científica "Educación y Ciencia" 3 (89-94) setiembre de 2007

Gagné, R. (1979). Principios básicos del aprendizaje para la instrucción. Edit. Diana, México.

Gutiérrez F., García Madruga, Elosúa y Otros (2002) Memoria operativa y comprensión lectora: algunas cuestiones básicas. Acción Psicológica.

Jiménez, J y Ortiz, M. (2000). Conciencia fonológica y aprendizaje de la lectura: teoría, evaluación e intervención. Madrid: Síntesis.

Luria, A. (1974). El cerebro en acción. Barcelona: Fontanella.

Sánchez, H. y Reyes, C. (2006) Metodología y diseño de la investigación científica. Lima: Editorial Visión Universitaria.

Whittaker, J. (1988). La psicología social en el mundo de hoy. Mexico: Edit. Trillas, 510pp.

Yarlequé, L. (1998). Psicología Evolutiva y Pedagógica. 2da. Edición. Huancayo: UNCP.

Yarlequé, L; Javier, L; y Monroe, J. (2002). Aprendizaje y Educación. Huancayo: Ediciones Omega. 
